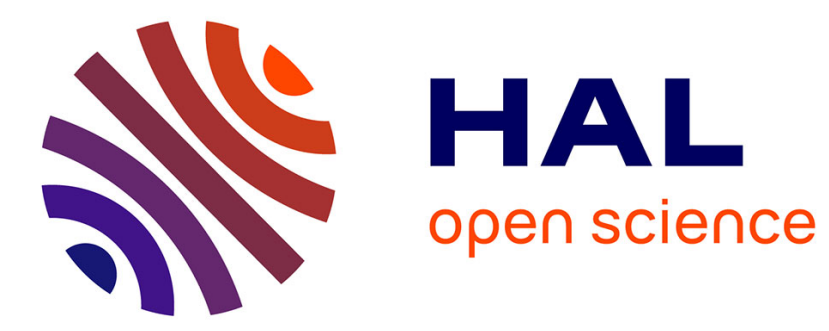

\title{
Enregistrement numérique direct du champ magnétique terrestre
}

\author{
R. Schlich
}

\section{To cite this version:}

R. Schlich. Enregistrement numérique direct du champ magnétique terrestre. Revue de Physique Appliquée, 1970, 5 (1), pp.153-158. 10.1051/rphysap:0197000501015300 . jpa-00243351

\section{HAL Id: jpa-00243351 https://hal.science/jpa-00243351}

Submitted on 1 Jan 1970

HAL is a multi-disciplinary open access archive for the deposit and dissemination of scientific research documents, whether they are published or not. The documents may come from teaching and research institutions in France or abroad, or from public or private research centers.
L'archive ouverte pluridisciplinaire HAL, est destinée au dépôt et à la diffusion de documents scientifiques de niveau recherche, publiés ou non, émanant des établissements d'enseignement et de recherche français ou étrangers, des laboratoires publics ou privés. 


\title{
ENREGISTREMENT NUMÉRIQUE DIREGT DU GHAMP MAGNÉTIQUE TERRESTRE
}

\author{
Par R. SCHLICH, \\ Centre National de la Recherche Scientifique, Institut de Physique du Globe de Paris, 94-Saint-Maur-des-Fossés.
}

\begin{abstract}
Résumé. - On propose un dispositif d'enregistrement numérique des variations du champ magnétique terrestre. Les signaux délivrés par trois capteurs, définissant en un point trois éléments du champ magnétique terrestre, sont échantillonnés simultanément, à intervalles de temps réguliers. L'instant du prélèvement est connu grâce à une unité " calendrier " et une horloge numérique. Les informations sont perforées sur ruban papier suivant le code IBM à 8 canaux ou suivant les normes internationales du code télex à 5 canaux et peuvent dans ce dernier cas être transmises ou reçues par radio.

L'exploitation des données enregistrées est immédiate, elle s'effectue automatiquement à l'aide d'ordinateurs.

Plusieurs dispositifs de ce type ont été mis en œuvre : à l'Observatoire magnétique de Dumont d’Urville en Terre Adélie, à l'Observatoire magnétique de Port-aux-Français dans l'archipel des Kerguélen et dans le cadre d'expériences entre points conjugués de France et d'Afrique du Sud.
\end{abstract}

La détermination de valeurs instantanées du champ magnétique terrestre, le calcul des valeurs moyennes horaires, journalières, mensuelles et annuelles à partir d'enregistrements analogiques, tels que les magnétogrammes La Cour ou Askania habituellement réalisés dans les observatoires magnétiques, constituent un travail particulièrement fastidieux. L'enregistrement des variations magnétiques sous forme numérique fournit une solution pratique à ce problème et augmente d'une façon considérable les possibilités d'analyse des perturbations enregistrées.

La nécessité de disposer de données magnétiques sous forme numérique a conduit de nombreux observatoires à mettre au point des dispositifs semi-automatiques permettant la numérisation des magnétogrammes La Cour. Ceci a été le cas pour les Observatoires américains du Coast and Geodetic Survey, pour nos Observatoires magnétiques des Terres Australes et Antarctiques Françaises [Schlich et Palomares, 1966], etc. En fait, jl s'agissait là d'une solution provisoire et pour supprimer toute intervention manuelle dans l'élaboration des données, nous avons entrepris l'étude et la réalisation d'un dispositif permettant l'enregistrement direct sous forme numérique de trois éléments indépendants du champ magnétique terrestre. Des ensembles visant le même objectif, mais différant tant par le choix des capteurs que par le mode d'enregistrement, ont été décrits par ailleurs. Signalons les plus connus, à savoir le dispositif d'enregistrement numérique réalisé à l'Observatoire magnétique de Kiruna en Suède [Gustafsson, 1967] et l'Observatoire magnétique automatique [Alldredge, 1960, 1963] qui, construit autour d'un magnétomètre à pompage optique, présente le grcs avantage de pouvoir s'affranchir de la servitude des mesures absolues, mais qui demeure encore d'un prix trop élevé et d'une complexité trop grande pour pouvoir être généralisé dans les Observatoires.

Finalement, la réalisation d'un dispositif d'enregistrement numérique des variations du champ magnétique terrestre pose essentiellement deux problèmes : celui du capteur et celui de l'enregistrement numérique proprement dit.

Le capteur. - En 1951, Dürschner réalisait à l'Institut de Physique du Globe de Paris un magnétomètre d'un type nouveau permettant l'enregistrement à distance des variations d'une composante horizontale du champ magnétique terrestre. Rappelons le principe de cet appareil : un équipage mobile constitué par un aimant et un miroir plan est suspendu entre deux bobines d'Helmholtz. Le miroir renvoie l'image donnée par une fente lumineuse sur deux cellules photoélectriques jumelles montées en opposition. Il est une position du spot pour laquelle (cellules également éclairées en principe) les deux forces électromotrices opposées sont égales. Une rotation de l'aimant à partir de la position correspondante provoque l'apparition d'un signal électrique aux bornes des cellules. Ce signal est amplifié et renvoyé dans les bobines d'Helmholtz de façon à compenser la variation de champ qui lui a donné naissance. L'aimant se trouve ainsi immobilisé dans une position de quasi-zéro et le courant nécessaire à la compensation est proportionnel à la variation de champ.

A la même époque [1951], un magnétomètre de ce type était réalisé à l'Observatoire magnétique de Kakioka. En 1956, Lebeau reprenant les travaux de Dürschner réalisait pour l'Observatoire magnétique du Dumont d'Urville en Terre Adélie un magnétomètre à contre-réaction de champ qui donna entière satisfaction et fonctionna pratiquement sans interrup- 
tion de 1957 à 1961. Ce résultat guida notre choix et c'est la raison pour laquelle nous avons adopté pour notre chaîne d'enregistrement le magnétomètre à contre-réaction de champ construit par Jolivet [1964] au Centre d'Études Géophysiques de Garchy. En fait, ces nouveaux magnétomètres se sont avérés extrêmement sensibles aux variations de température et leur utilisation en tant que variomètre d'observatoire n'a pas été immédiate. Il a fallu d'une part intervenir sur le magnétomètre lui-même et d'autre part réaliser une double enceinte amagnétique thermostatée permettant de maintenir la température constante à $0,2^{\circ} \mathrm{C}$ au niveau des capteurs.

Le dispositif d'enregistrement numérique. - On dispose en principe de trois capteurs définissant en un point le champ magnétique terrestre. On enregistre soit les composantes $X, Y, Z$ ou $H, D, Z$, soit de préférence, étant donné les difficultés rencontrées avec les magnétomètres Jolivet, les éléments $X, Y, F$ ou $H$, $D, F$. L'intensité du champ total $(F)$ est mesurée à l'aide d'un magnétomètre nucléaire.

Les signaux analogiques délivrés par les magnétomètres sont échantillonnés simultanément à intervalles de temps réguliers et sont enregistrés dans des mémoires capacitives. Un voltmètre numérique assure la conversion analogique-numérique. On obtient ainsi trois valeurs qui permettent le calcul du champ terrestre et de ses composantes à un instant déterminé. L'instant de la mesure est connu grâce à une unité « calendrier » et une horloge numérique. Les données, dates, heures, champ magnétique, sont perforées sur ruban papier.

La figure 1 représente le schéma synoptique du dispositif d'enregistrement numérique, il comprend :

- une unité de « mémorisation »,

- un sélecteur de voie,

- un convertisseur analogique-numérique,

- une unité « calendrier »,

- une horloge numérique,

- un standard de fréquence,

- une unité de codage et de programmation,

- un clavier pour entrée manuelle d'informations, - une perforatrice de bande.

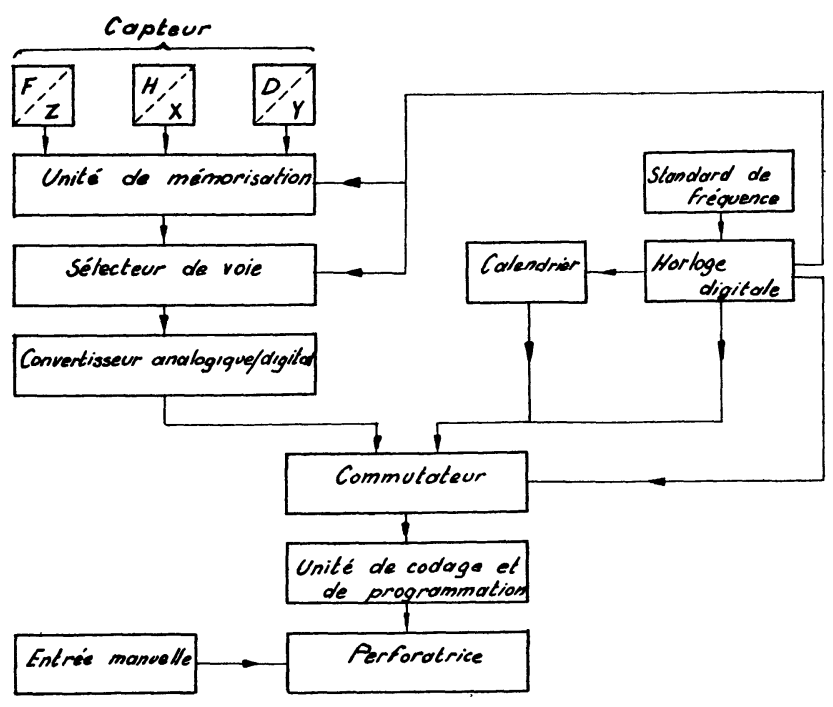

Fig. 1. - Schéma synoptique du dispositif d'enregistrement numérique des variations du champ magnétique terrestre.
Pour permettre le calcul d'une composante quelconque du champ magnétique terrestre ou certaines études, celles par exemple de la direction et de la polarisation du vecteur perturbation, il est indispensable que la mesure des trois composantes du champ se fasse à un même instant. C'est cela qui nous a conduits à envisager une unité dite de "mémorisation »; elle est constituée essentiellement par trois capacités au polystyrène de $2 \mu \mathrm{F}$ chacune. Ces capacités étant en liaison directe avec les signaux à mesurer, leurs charges suivent les variations du champ magnétique terrestre; à l'instant de la mesure, elles sont déconnectées de leur source respective et sont reliées successivement au convertisseur analogique-numérique. L'exploration des trois canaux se fait à raison de deux voies par seconde. Le convertisseur est utilisé avec une impédance d'entrée supérieure à $1000 \mathrm{M} \Omega$; dans ces conditions, la perte de charge pendant le temps nécessaire à la mesure est négligeable, du moins elle est inférieure à la précision du convertisseur. Les trois mesures étant effectuées, les capacités sont à nouveau connectées aux magnétomètres, et ainsi de suite. Les mesures peuvent être faites, au choix, toutes les 3,5 , 15,30 ou $60 \mathrm{~s}$. Suivant le type d'étude entreprise ou le niveau de l'agitation magnétique, un commutateur à 5 positions permet une sélection instantanée de la cadence d'échantillonnage. Il est possible, par ailleurs, après avoir sélectionné une voie déterminée, d'effectuer manuellement une mesure à un instant quelconque; ceci permet de vérifier, avant la mise en marche de l'installation, le fonctionnement des différentes voies et facilite les manipulations lors de la mise en station des magnétomètres et les opérations d'étalonnage. Les ordres d'échantillonnage et d'exploration des trois mémoires sont donnés par des impulsions prélevées au niveau de l'horloge.

Le convertisseur est un voltmètre numérique à 5 chiffres pouvant mesurer des tensions allant de $20 \mu \mathrm{V}$ à 1599,9 . Seules les deux premières gammes $(0$ à $0,15999 \mathrm{~V}$ et 0 à $1,5999 \mathrm{~V}$ ) ont une impédance d'entrée suffisamment élevée $(>1000 \mathrm{M} \Omega$ ) pour permettre la lecture de capacités avec une précision de $0,02 \% \pm$ une unité; on utilisera par conséquent nécessairement l'une de ces sensibilités. La tension mesurée est affichée avec son signe sur la face avant du voltmètre; en fait, les trois mesures apparaissent successivement sur le cadran lumineux du voltmètre, la troisième valeur demeurant affichée jusqu'au début du cycle suivant; elles sont perforées en séquence, nous verrons plus loin les détails de cette opération.

L'horloge numérique délivre les différentes impulsions nécessaires au fonctionnement de cette chaîne de mesure; elle permet par ailleurs de situer dans le temps les valeurs de champ mesurées. L'heure, les minutes et les secondes sont affichées en permanence sur un ensemble de cadrans. Une unité complémentaire dite « calendrier » complète cette information; la progression des jours est commandée par l'horloge, le quantième du mois apparaissant également sur deux cadrans lumineux; le mois et l'année sont présélectionnés manuellement à l'aide de 4 commutateurs, deux pour les mois et deux pour l'année; on affiche 01 pour le mois de janvier, etc., et les deux derniers chiffres correspondant à l'année. Un commutateur à 4 positions commande la remise à zéro automatique du calendrier après $28,29,30$ ou 31 jours; suivant la longueur du mois, on choisira l'une ou l'autre de ces 
positions. Cette information de date et d'heure, appelée aussi « adresse», est perforée aux heures rondes préalablement aux valeurs de champ magnétique correspondantes. Suivant la cadence adoptée pour les mesures, chaque adresse sera séparée par 1200,720 , 240,120 ou 60 groupes de valeurs. L'adresse pourra également être prélevée à un instant quelconque par commande manuelle; ainsi, chaque début de bande comportera une adresse, cette information étant indispensable pour le traitement ultérieur des données recueillies.

L'horloge peut être pilotée directement par le courant alternatif $50 \mathrm{~Hz}$ du secteur; en fait, on utilise un standard de fréquence qui assure par jour une précision supérieure à la seconde, on évite ainsi la nécessité de recaler fréquemment le système de mesure; enfin, pour les échantillonnages à cadence élevée, il est indispensable de connaître l'heure avec précision. Il y a lieu d'attirer l'attention sur le fait que l'exploration et la perforation de l'adresse nécessitent environ $1 \mathrm{~s}$, ceci conduit, aux heures rondes, à différer la perforation des informations « champ magnétique » d'un temps correspondant, d'où perte de charge plus importante au niveau des capacités mémoires. Afin d'éviter cet inconvénient, tout en respectant l'homogénéité des intervalles séparant deux mesures consécutives, on préfère décaler systématiquement d'une seconde l'instant des prélèvements. Les trois mesures adressées $00 \mathrm{~h} 00 \mathrm{mn} 00 \mathrm{~s}$ correspondent en fait à l'instant $00 \mathrm{~h} 00 \mathrm{mn} 01 \mathrm{~s}$ du système, etc. On pourra tenir compte de cette différence en décalant l'horloge d'une seconde par rapport au temps universel : on respectera ainsi la correspondance entre l'adresse et l'instant de la mesure en temps universel; par ailleurs, on rétablira aussi au niveau de l'enregistrement la correspondance entre l'adresse, l'instant effectif de la mesure et l'heure universelle.

Les données numériques, valeurs de champ magnétique ou adresse suivant le cas, c'est-à-dire suivant la position d'un commutateur commandé par l'horloge, sont codées puis perforées sur bandes papier. On a adopté les normes internationales du code télex à 5 canaux, tous les ordres de service ont été prévus et l'information peut être transmise et reçue par radio; cette possibilité est surtout intéressante dans le cas d'observatoires magnétiques lointains. En fait, moyennant quelques modifications mineures, il est possible de perforer les informations en code IBM à 8 canaux. L'unité de codage comporte un bloc de programmation qui tient compte de la longueur variable des mots suivant qu'il s'agit d'une information issue du voltmètre numérique ou du calendrier; enfin, c'est à ce niveau que sont introduits les caractères fixes nécessaires à l'identification des groupes et à la transmission par radio dans le cas du code télex.

L'adresse comporte 14 caractères, les deux premiers correspondent aux deux derniers chiffres de l'année, les deux suivants au mois, et ainsi de suite pour le jour, l'heure, les minutes et les secondes. La fin du mot est marquée par les instructions « retour chariot» et « avance papier ». L'information champ magnétique comprend 24 caractères dont 18 réservés aux mesures proprement dites; les deux premières mesures sont suivies chacune de deux caractères fixes, le premier correspond à un espace, le deuxième au code « chiffre » nécessaire dans le cas du code télex pour différencier les chiffres des lettres; dans le cas du code IBM à 8 canaux, cette instruction est remplacée par un deuxième espace. Comme précédemment, la fin du mot est marquée par les instructions « retour chariot» et « avance papier ». Sur la bande perforée les caractères se succèdent dans l'ordre suivant :

A A M M J H H m m s G L

S Z Z Z Z Z E S X X X X X E e S Y Y Y Y Y G L S Z Z Z Z Z E e, etc.

A A

M M

$\mathrm{JJ}$

$\mathrm{H} \mathrm{H}$ année (deux derniers chiffres)

mois

jour

$\mathrm{m} \mathrm{m}$

heure

minutes

s s secondes

C retour chariot

L avance papier

$\mathrm{S} \quad$ signe + ou -

E espace

e code « chiffre » ou espace suivant le code utilisé

\section{$\mathrm{ZZZZZ}$}

X X X X X champ magnétique

Y Y Y Y Y

Enfin, il faut signaler qu'il est possible d'ajouter manuellement sur la bande, à un instant quelconque, des informations supplémentaires, comme par exemple le nom de l'observatoire, les composantes enregistrées, l'ordre dans lequel elles sont perforées sur la bande, les valeurs d'échelle, etc.

Traitement des informations numériques. - La bande perforée telle qu'elle a été décrite au paragraphe précédent peut, moyennant un programme approprié et un calculateur équipé d'un lecteur de ruban perforé, être utilisée directement; il sera en particulier possible d'entreprendre tous les calculs généralement effectués à partir des données d'observatoires magnétiques.

En fait, on préfère, pour pouvoir accéder plus facilement aux gros calculateurs, transcrire l'ensemble des informations recueillies sur bande magnétique et entreprendre à partir de la bande ainsi créée l'élimination des valeurs aberrantes et le calcul des valeurs instantanées des composantes du champ magnétique terrestre.

Nous n'examinerons pas ici le détail de ce traitement, mais remarquons simplement que l'identification des mots pourra se faire par la position du caractère « fin de mot» ou encore par la nature du premier caractère qui, dans le cas d'une « adresse », sera nécessairement un chiffre supérieur ou égal à 6 et dans le cas d'une information " champ magnétique » sera toujours, étant donné les caractéristiques du convertisseur analogique-numérique, un signe plus ou moins suivi d'un zéro ou de l'unité. Les valeurs ou mesures erronées pourront être détectées et éliminées en examinant les différences premières et secondes formées à partir des valeurs successives enregistrées.

Exemples d'installations réalisées. - Plusieurs installations de ce type ont été mises en œuvre : en 1965, à l'Observatoire magnétique de Dumont d'Urville en Terre Adélie [Schlich, 1965], en 1967 à l'Observatoire magnétique de Port-aux-Français dans l'archipel des Kerguélen et enfin, en France à la station du Hurou- 
TABLEAU I

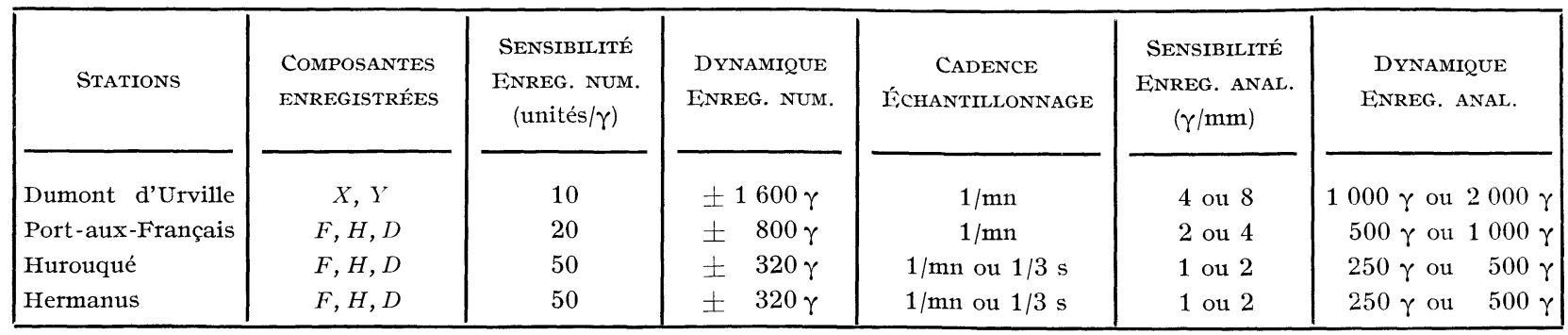

qué dans les Landes et en Afrique du Sud à l'Observatoire d'Hermanus (1965-1966) dans le cadre d'un programme d'expériences entre points conjugués. Dans les deux premiers cas, il s'agissait essentiellement de compléter par un enregistrement numérique les enregistrements analogiques classiques réalisés dans ces observatoires à l'aide de magnétographes à enregistrement photographique et apporter ainsi une solution pratique aux problèmes posés par le calcul des valeurs moyennes horaires du champ magnétique terrestre et permettre éventuellement la transmission par radio des données recueillies en ces stations lointaines. Pour les installations réalisées en France et en Afrique du Sud, il s'agissait de mettre en œuvre des équipements identiques permettant de comparer facilement les perturbations magnétiques enregistrées en deux régions magnétiquement conjuguées.

Précisons que les enregistrements numériques sont toujours complétés par un enregistrement analogique continu réalisé à l'aide d'un enregistreur potentiométrique à 3 voies, ceci afin de visualiser directement les perturbations magnétiques enregistrées. Les caractéristiques des différents montages mis en œuvre sont résumées dans le tableau I.

La figure 2 donne le schéma du montage tel qu'il a été réalisé dans nos différentes stations et la figure 3 représente le système complet avec le dispositif de numérisation, la perforatrice de ruban et l'enregistreur potentiométrique.

Pour illustrer les possibilités offertes par un tel équipement, et à titre d'exemple, on reproduit quelques extraits d'enregistrements numériques que l'on compare aux enregistrements analogiques correspondants. La figure 4 représente pour la station du Hurouqué dans les Landes, l'enregistrement réalisé le 29 août 1966. L'enregistrement numérique a été res-

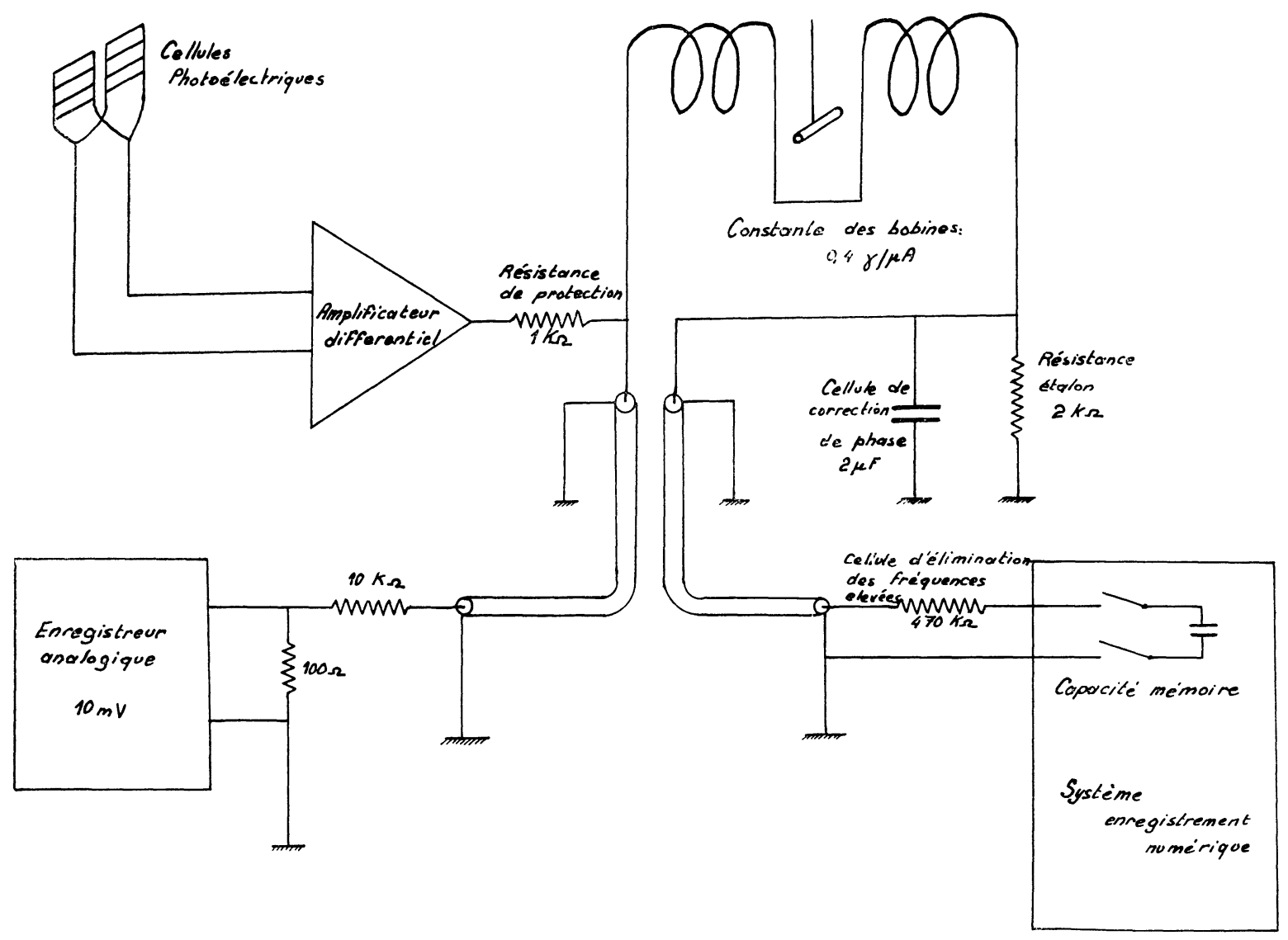

FIG 2. - Schéma de montage du dispositif d'enregistrement numérique et analogique. 


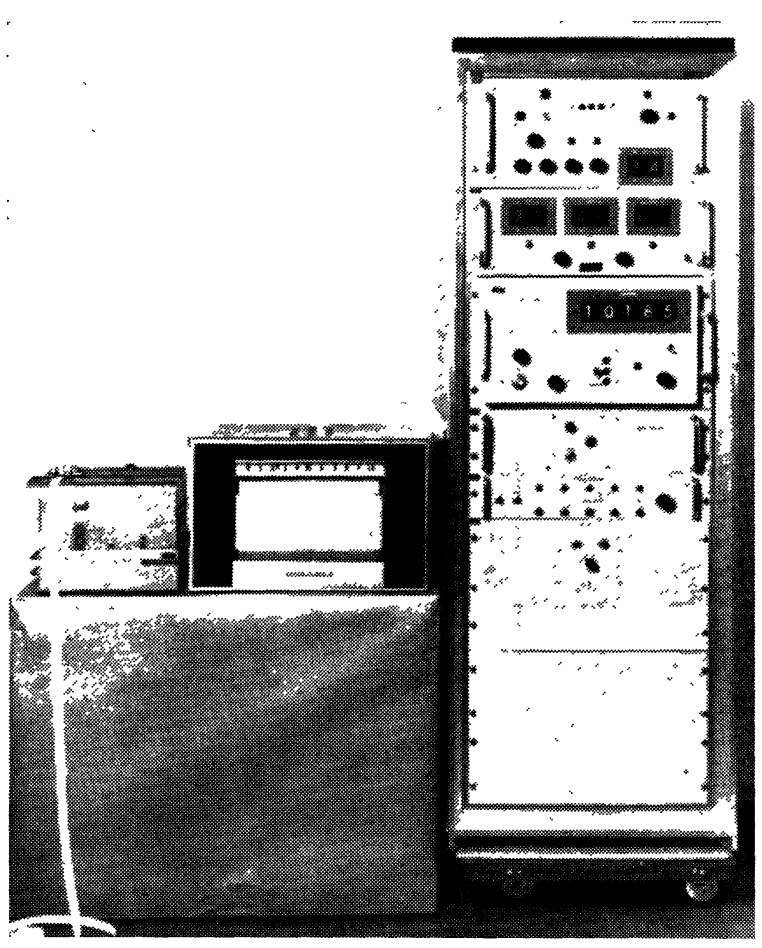

FIG. 3. - Dispositif d'enregistrement numérique des variations du champ magnétique terrestre.

titué à l'échelle de l'enregistrement analogique pour faciliter la comparaison; entre 13 et 14 heures, on a pointé deux mesures par mn et entre 14 et 17 heures une seule mesure par $\mathrm{mn}$. La figure 5 représente, pour le même jour, restitué à partir de l'enregistrement numérique, le début brusque enregistré entre 13
HUROUQUE 29-8-66

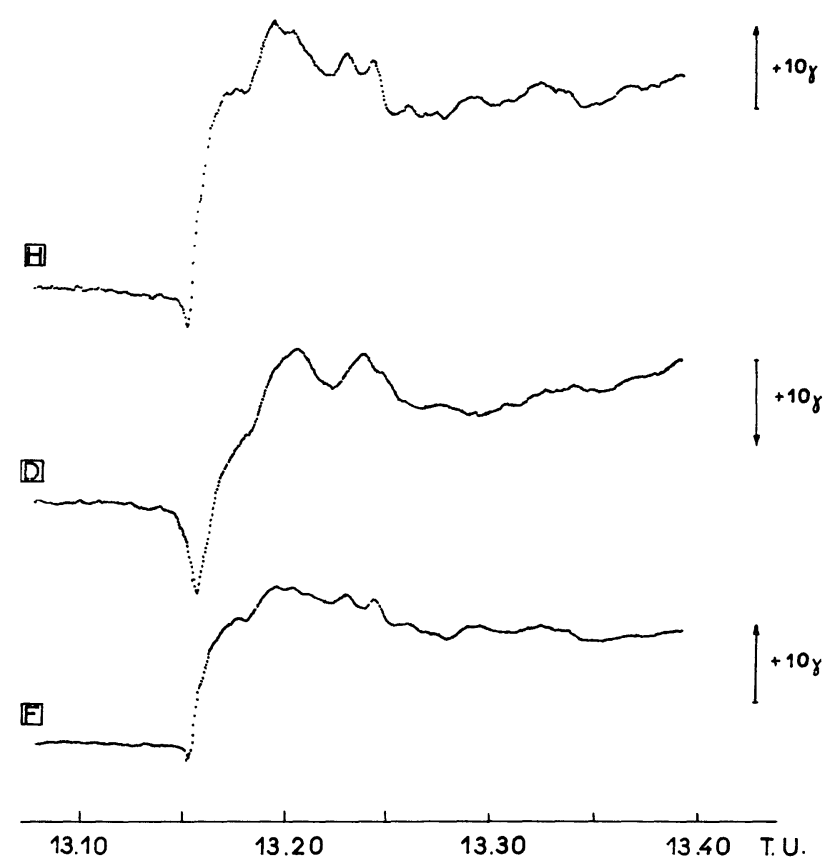

FIG. 5. - Restitution à partir des informations numériques du début brusque enregistré au Hurouqué le 29 août 1966.

et 14 heures T.U., l'échelle des ordonnées a été multipliée par deux et l'échelle des temps par 6 ; la restitution est faite dans ce cas en pointant toutes les valeurs numériques enregistrées, c'est-à-dire une lecture toutes les $3 \mathrm{~s}$.
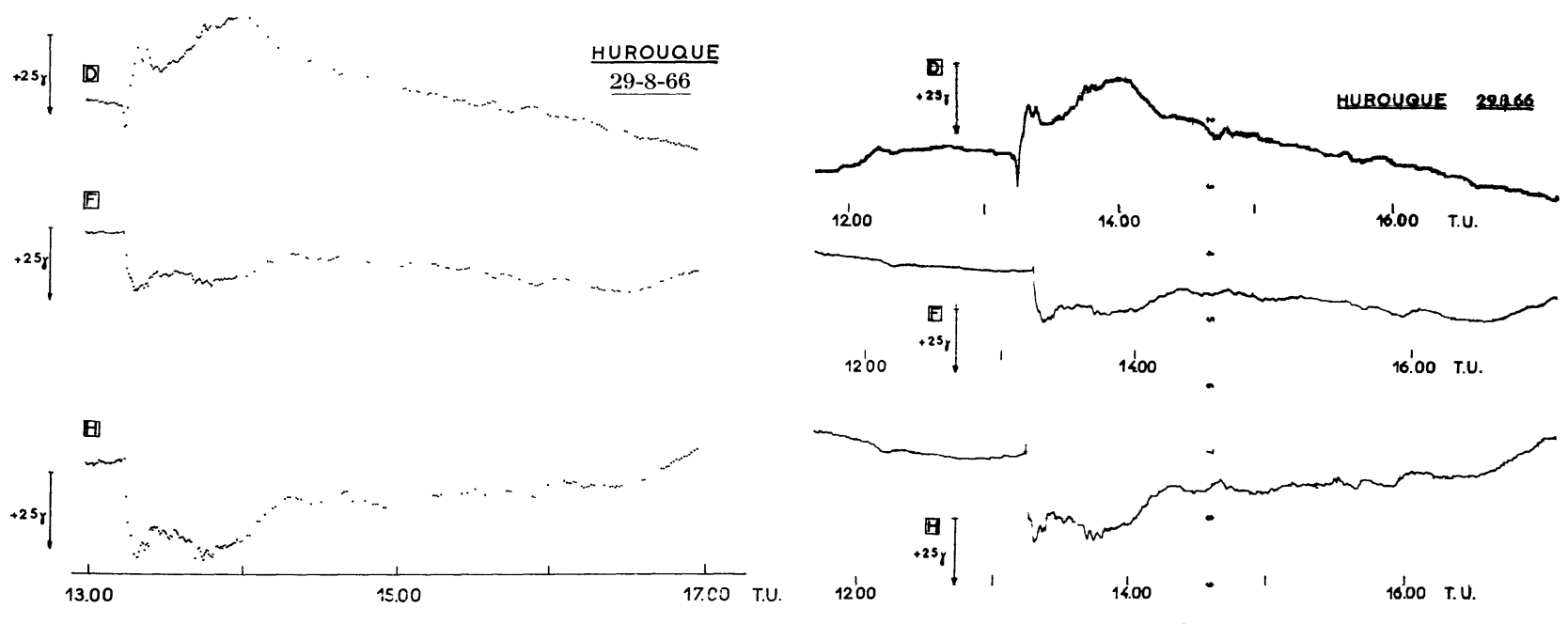

a

b

FIG. 4. - Exemple d'enregistrement restitué à partir des informations numériques.

Comparaison avec les enregistrements analogiques correspondants.

\section{BIBLIOGRAPHIE}

ALLDREDGE (L. R.), A proposed automatic standard magnetic observatory, J. Geophys. Res., 1960, 65, 11, $3777-3786$.
ALLDREDGE (L. R.) et SALDUKas (I.), An automatic standard magnetic observatory, U.S. Department of Commerce, Coast and Geodetic Survey, août 1963. 
DÜRSCHNER (H.), Enregistrement à distance des variations du champ magnétique terrestre, Ann. de Géophys., 1951, 7, 199-207.

Gustafsson (G.), Thunberg (A.) et HeikKIIA (K. E.), A system for automatic collection and processing of standard magnetometer data, and the calculation of their power spectrum, Contribution from Kiruna Geophysical Observatory of the Royal Swedish Academy of Science, Contract No. AF 61 (052)-237, april 1963.

GustafsSON (G.), A system for automatic collection of geomagnetic data in digital form, Contribution from Kiruna Geophysical Observatory of the Royal Swedish Academy of Science, Contract No. AF 61 (052)-237, february 1967.

Hultovist (B.), Thunberg (A.), LINDELI (S.) et HEIKKILA (K. E.), An automatic digital recording and reducing system for geomagnetic standard data, Arkiv for geofysik, 1962, 4, 1-23.
JOLIVET (A.), Magnétomètre antivibratoire à immersion et contre-réaction de champ, Diplôme d'Études Supétieures de Sciences Physiques, Paris, 1964.

LEBEAU (A.), Upper Atmosphere Investigations, French Antarctic Expedition, Dumont d'Urville Station, Terrestrial Magnetism, Symposium on Antarctic Research Wellington, New Zealand, 12-14, 1958.

Mem. of the Kakioka Magnetic Observatory, 1951, 6, 64.

ScHIICH (R.), Rénovation de l'Observatoire magnétique de Dumont d'Urville, Bulletin d'information, 1964, 16, 7-13.

Schlich (R.) et Palomares (M.), Traitement semiautomatique d'enregistrements analogiques, application aux magnétogrammes, Ann. de l'Institut de Physique du Globe de Paris, 1966, XXXIV, 121-147. 